TRADUÇÕES - TRANSLATIONS

\title{
Georges Canguilhem et la construction du domaine de la Santé collective brésilienne ${ }^{1}$
}

\author{
José Ricardo de Carvalho Mesquita Ayres \\ Professor Titular do Departamento de Medicina Preventiva - Faculdade de Medicina USP \\ ircayres@usp.br
}

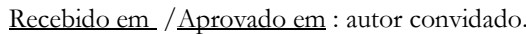

Como citar este artigo: Ayres, José Ricardo de Carvalho Mesquita. "Georges Canguilhem et la construction du domaine de la Santé collective brésilienne”. Intelligere, Revista de História Intelectual, São Paulo, v. 3, n 1 [4], p. 144-161. 2017. ISSN 2447-9020. Disponível em <http://revistas.usp.br/revistaintelligere>. Acesso em dd/mm/aaaa.

Résumé: L'épistémologie historique a joué un rôle remarqué dans le développement de la Santé collective ${ }^{2}$ brésilienne. Née comme un mouvement simultanément académique, de recherche de nouvelles bases conceptuelles pour un domaine scientifique engagé dans les causes sociales, et politique, de résistance contre la dictature civile et militaire implantée au Brésil en 1964, ledit Mouvement de réforme sanitaire brésilienne a trouvé dans l'épistémologie historique française un allié puissant. Cet article cherche à revisiter les principaux traits de cette relation, en se focalisant principalement sur les travaux inauguraux de Sergio Arouca et Cecilia Donnangelo et la théorie du processus de travail dans la Santé, développée au sein du Département de médecine préventive de la Faculté de médecine de l’Université de São Paulo. La discussion est centrée sur la manière selon laquelle, d'un côté, les concepts philosophiques de Canguilhem, comme le caractère normatif de la vie et de sa connaissance, la discontinuité qualitative entre les phénomènes normaux et pathologiques, la définition de la santé orientée par la notion de valeur et, d'un autre côté, la méthodologie historique de Canguilhem, focalisée sur le développement rationnel des concepts comme noyau de la recherche historico-épistémologique, la reconnaissance des influences "externes" sur les développements scientifiques, comme les conditions sociales et technologiques, et le rôle positif attribué aux obstacles, aux défaillances et accidents des progrès scientifiques, ont toutes été cruciaux pour promouvoir l'articulation entre les intérêts politiques et académiques du Mouvement. Ils sont encore des éléments de défi pour les réflexions historiques et philosophiques de la Santé collective.

Mots-clefs: Santé publique/histoire; Médecine préventive; Médecine sociale; Épistémologie; Santé collective; Georges Canguilhem.

\footnotetext{
${ }^{1}$ Travail en relation au projet "Histoire de la Santé collective dans l'État de São Paulo: émergence et développement d'un domaine du savoir et des pratiques" (FAPESP 2013/12137-0) e présenté au colloque: “Canguilhem, l'histoire et les historiens", organisé par le Département d’histoire de la Faculté de philosophie, lettres et sciences humaines de la Université de São Paulo en décembre 2015. Publié sous le titre "Georges Canguilhem e a construção do campo da saúde coletiva brasileira” dans Intelligere, Revista de História Intelectual, São Paulo, v. 2, n. 1 [2], p. 139-155. 2016.

${ }^{2}$ Note de traduction: L'expression "Santé collective" et le choix de l'adjectif "collectif" qui accompagne le terme "santé" est une invention brésilienne pour qualifier la moderne Santé publique brésilienne en réaction aux anciens concepts de la Santé publique traditionnelle.
} 


\title{
Georges Canguilhem and the development of Brazilian Public Heatlh field
}

\begin{abstract}
Historical epistemology has played an important role in the development of modern Brazilian Public Health or "Saúde Coletiva" (Collective Health). Born as an academic search for new conceptual foundations of a social committed field of scientific knowledge, as well as a social political movement against civil-military dictatorship implanted in Brazil in 1964, the so called Brazilian Sanitary Reform Movement found in the French historical epistemology, particularly in the works of Georges Canguilhem, a powerful ally. This paper aims to revisit the main features of this relationship, focusing in particular the inaugural works of Sergio Arouca and Cecília Donnangelo and the Health Work Process Theory as developed in the Department of Preventive Medicine of the Medical School of the University of São Paulo. The discussion is centered in the way Canguilhem's philosophical concepts, such as the normative character of life and of its knowledge, the qualitative discontinuity between normal and pathological phenomena and the value oriented definition of health on one hand, and on the other hand, Canguilhem's historiographical method, focused on the rational development of concepts as the core subject of the historical-epistemological research, the acknowledge of "external" influences over scientific developments, such as social and technological conditions, and the positive role attributed to obstacles, failures and accidents in the progress of a scientific discipline were all crucial to promote the intertwining of the political and academic goals of the Movement and still remain a challenging element for the development of the philosophical and historical reflections of the field of "Saúde Coletiva".
\end{abstract}

Keywords: Public Health/History; Preventive Medicine; Social Medicine; Epistemology; Collective Health; Georges Canguilhem.

\section{Canguilhem par un médecin sanitaire, quelques notes introductives}

Cet article n'est pas un travail d'historien, mais peut être utile aux historiens. Je m’explique. Je suis médecin sanitaire et professeur en Santé collective. Parmi les diverses activités embrassées par un ample et divers domaine comme celui-ci, je me suis consacré aussi aux études de ses racines historiques et de ses bases épistémologiques et conceptuelles. Comme d'autres dans mon domaine, je me suis aventuré sur des sentiers interdisciplinaires - non sans le risque toujours impliqué par ces aventures - qui ont fini par m’amener à l'épistémologie historique. Cependant, je suis arrivé là non pas pour des intérêts strictement historiographiques ou philosophiques, mais pour voir dans ce dialogue les possibilités d'une meilleure compréhension de ce que nous faisons et ce que nous ne parvenons pas à faire dans la Santé collective et penser des stratégies pour la perfectionner aussi bien dans ses investissements critiques caractéristiques de la science et les techniques de la santé, que dans ses engagements pratiques et tâches instrumentales ${ }^{3}$.

\footnotetext{
${ }^{3}$ J. Silva Paim e Naomar de Almeida Filho, A crise da Saúde Pública e a utopia da Saúde Coletiva LLa crise de la Santé publique et l'utopie de la Santé collective] (Salvador: Casa da Qualidade, 2000).
} 
Sur la base de cette expérience, je cherche ici à récupérer une partie du dialogue établi entre la Santé collective, dans son processus de constitution comme domaine académique, et l'épistémologie historique. J'espère fournir quelques indices sur combien, dans l'histoire des effets $^{4}$ de la tradition discursive de l'épistémologie historique française, on compte une lecture influente de Georges Canguilhem (1904-1995) dans ce domaine, même si les contributions de ce philosophe et médecin sont loin d'avoir été incorporées ici de façon radicale aux concepts et aux pratiques $^{5}$.

Dans ce sens, pour que je sois mieux compris, il faut aider le lecteur qui n'est pas familiarisé avec la Santé collective. Cette tâche n’est pas si simple, mais peut-être de manière bien synthétique on peut la définir comme un mouvement d'idées et de pratiques qui émergent au Brésil dans les années 1970. Ayant comme marque institutionnelle la création, en 1979 de l'ABRASCO (alors Association Brésilienne de Post-graduation en Santé collective, aujourd'hui simplement Association Brésilienne de Santé Collective), ce domaine s'est développé en étroite relation avec le processus appelé Réforme sanitaire et avec la création du Système Unique de Santé (SUS) en 1988 . En synthèse, d'une forme intégrée à la lutte contre la dictature civil-militaire d'alors et au processus de démocratisation du pays, le domaine de la Santé collective cherchait à construire des savoirs et des pratiques activement intéressés par les déterminants socioculturels et économiques des processus de santé-maladie-soin, tout en articulant ses constructions scientifiques et ses propositions techniques en santé avec les compromis de l'émancipation politiques des sujets, de la démocratie et la justice sociale.

La lecture de Georges Canguilhem, spécialement son texte de 1943: Le normal et le pathologique, ${ }^{7}$ a accompagné le projet académique de la Santé publique depuis le début, devenant une référence des œuvres fondatrices de ce domaine, ${ }^{8}$ en particulier dans la thèse de doctorat de Sergio Arouca (1941-2003), Le Dilemme préventiviste: contribution pour la comprébension et la critique de la médecine préventive 9 et celle de habilitation de Cecilia Donnangelo (1940-1983), Médecine et structure sociale: le domaine d'émergence de la médecine communautaire ${ }^{10}$. Le type d'approximation critique aux sciences médicales et à certaines conceptions et pratiques de la santé développée par Canguilhem s'est montrée assez intéressant dans la perspective des impulsions reconstructives qui animaient la Santé collective depuis ses origines. Au long de près de quarante ans d'existence de ce domaine, comme on pouvait s'y attendre, son influence s'est diluée et diversifiée, sans perdre pour cela sa pertinence comme cela peut être attestée par les références déjà citées. Entre les divers destins de la lecture de son œuvre, je remarquerai le vif intérêt des professeurs du Département de Médecine Préventive de la Faculté de Médecine de l’Université de São Paulo, auquel se trouve liée ma propre

${ }^{4}$ H.G. Gadamer, Verdade e método: traços fundamentais de uma bermenêutica filosófica [Vérité et méthode: traits fondamentaux d'une herméneutique philosophique] (Petrópolis: Vozes, 2002).

${ }^{5}$ K.R. Camargo Jr., “As armadilhas da concepção positiva de saúde” [Les pièges de la conception positive de la sante], Physys: Revista de Saúde Coletiva, [vol.] 17, 1 (1991): 63-76.

${ }^{6}$ J. S. Paim, Reforma Sanitária Brasileira: contribuição para a compreensão e crítica [Réforme sanitaire brésilienne: contributions à la comprébension et critique] (Salvador: EDUFBA/Rio de Janeiro: Editora Fiocruz, 2006).

${ }^{7}$ Georges Canguilhem, O normal e o patológico patológico [Le normal et le pathologique] (Rio de Janeiro: Forense Universitária, 2015).

${ }^{8}$ Cf. Maria Thereza Ávila Dantas Coelho e Naomar de Almeida Filho, "Normal-patológico, saúde-doença: revisitando Canguilhem" ["Normal-pathologique, santé-maladie: revisitant Canguilhem"], Physis: Revista de Saúde Coletiva, [vol.] 9, 1 (1999): 13-36. Cet article soutient la position ici défendue à propos de la forte présence de Canguilhem (et Foucault) dans les œuvres fondatrices de la Santé collective. Néanmoins, quoique ce ne soit pas le point central de cet article, ses auteurs tendent à trouver qu'une telle importance aurait été un phénomène presque fortuit à une identification équivoque des œuvres de ces philosophes à une épistémologie de gauche. Sans entrer dans fond du débat (les œuvres de Canguilhem ont-elles été ou non vue à l'époque comme "de gauche" ? Pourraient-elles ou non recevoir cette classification ?), je n'accompagne pas les auteurs dans cette interprétation et je pense que le dialogue avec Canguilhem a été une clé pour créer le lien productif que la Santé collective est arrivé à établir entre le plan politique, des mouvements sociaux et des services d'un côté, et de la communauté scientifique de la médecine et de la santé publique, d'un autre côté. Et cela moins par le supposée articulation de ces auteurs avec quelque cadre théorique de gauche, que parce qu ils ont fourni des recours conceptuels effectifs pour la relation de l' "interne" et de l' "externe" dans les sciences de la santé, comme je chercherai à le montrer dans l'article.

${ }^{9} \mathrm{~S}$. Arouca, O dilema preventivista: contribuição para a compreensão e crítica da Medicina Preventiva [Le dilemme preventiviste: contribution pour la comprébension et la critique de la Médecine préventive] (Tese de doutorado, Universidade Estadual de Campinas, 1975).

${ }_{10}$ M. C. F Donnangelo, Medicina e estrutura social: o campo de emergência da Medicina Comunitária [Médecine et structure sociale: le domain d'emergence de la Médecine communautaire] (Tese de Livre-Docência , USP, 1976), 95-124. 
formation et production en Santé collective. La construction du cadre de la Théorie du processus de travail en Santé collective ${ }^{11}$ s'est divisée en une série de lignes et de projets d'investigation, constituant ce qui peut être considéré comme une certaine "école de pensée" parmi les diverses qui composent l'ample et pluriel domaine de la Santé collective brésilienne ${ }^{12}$. Je pense que ma participation de ce côté me permettait d'identifier avec une certaine clarté ce qu'a signifié Canguilhem dans cette trajectoire et les défis actuels de cette référence toujours vivante.

\section{Canguilhem et les origines de la Santé collective.}

Les œuvres de Canguilhem ont commencé à être plus amplement divulguées en portugais à partir de la fin des années 1970. En 1978 est divulguée la première édition de: Le normal et le pathologique ${ }^{13}$. Idéologie et rationalité dans l'histoire des sciences de la vie fut le second texte à circuler en portugais, mais publié au Portugal par les éditions 7014. Edité en portugais en 1981, la circulation de cette œuvre a été plus tardive et moins expressive dans la Santé collective, même si elle était lue par quelques chercheurs du domaine qui avaient accès à l'œuvre originale en français ${ }^{15}$. Après il s'en est suivi un long interrègne et c'est seulement dans les années 2000 que des éditions d'œuvres inédites de Canguilhem en portugais circulent de nouveaur, comme les Ecrits sur la médecine ${ }^{16}$, Études d'histoire et de philosophie des sciences: concernant les vivants et la vie ${ }^{17}$ et La connaissance de la vie. ${ }^{18}$

On doit signaler qu'avant la circulation de ces éditions, la revue Tempo Brasileiro avait déjà publié, début 1972, un numéro entièrement dédié à l'épistémologie historique française ${ }^{19}$, qui proposait deux traductions de textes de Bachelard, deux de Canguilhem, un de Foucault et un de Jacques Alain Miller. Les textes de Canguilhem inclus dans cette publication collective ont aussi été abondamment cités au sein de la Santé collective, notamment L'objet de l'bistoire des sciences ${ }^{20}$. Ce travail est cité par Sergio Arouca dans sa thèse parmi d'autres références à des œuvres de penseurs que seraient, selon lui, en train de favoriser des mouvements rénovateurs dans la Médecine Préventive, en rendant possible de penser les sciences sous une nouvelle perspective, plus adéquates aux références critiques marxistes - adéquation également valorisée par Althusser. ${ }^{21}$

À la suite de celle de Bachelard, l'histoire épistémologique de Canguilhem rompait avec le dogme du progrès continu et de l'immanence logique de la connaissance, elle incorporait la matérialité technique à la compréhension des possibilités heuristiques des sciences et, plus spécialement, considérait l'aspect idéologique comme un élément nécessaire pour élucider la construction de concepts (en considérant cependant l'idéologie dans un sens divergeant sur beaucoup d'aspects de celle qu'elle assumait dans la tradition marxiste). Avec cela, l'œuvre de Canguilhem offrait un support consistant pour les élans rénovateurs d'esprit politico-émancipateur

\footnotetext{
11 Ricardo Bruno Mendes-Gonçalves, Práticas de saúde e tecnologias: contribuição para a reflexão teórica [Pratiques en santé et technologies: contribution pour une réflexion théorique] (Brasília: OPS, 1988).

${ }^{12}$ R. B. Mendes-Gonçalves, Prefácio a José Ricardo de C. M. Ayres, Epidemiologia e emancipação [Preface a Epidémiologie et émancipation ] (São Paulo: Hucitec, 1995), 13-20.

${ }^{13}$ G. Canguilhem, O normal e o patológico (Rio de Janeiro: Forense Universitária, 1978).

${ }^{14} \mathrm{G}$. Canguilhem. Ideologia e racionalidade nas ciências da vida (Lisboa: Edições 70, 1981)

15 R. L. Novaes, A saúde e os conceitos [La santé et les concepts] (Dissertação de mestrado, Universidade de São Paulo, 1976); R. L. Novaes, Saúde e seu desenvolvimento bistórico: aspectos críticos [Santé et son développement historique: aspects critiques] (São Paulo, Departamento de Medicina Preventiva da FMUSP/Mimeo, 1973) e H. M. D. Novaes, A puericultura em questão [La puériculture en question] (Dissertação de mestrado, Universidade de São Paulo, 1979).

${ }^{16} \mathrm{G}$. Canguilhem, Escritos sobre a medicina. (Rio de Janeiro: Forense-Universitária, 2005).

${ }_{17} \mathrm{G}$. Canguilhem, Estudos de história e de filosofia das ciências: concernentes aos vivos e à vida (Rio de Janeiro: Forense Universitária, 2012).

${ }^{18}$ G. Canguilhem, O conhecimento da vida (Rio de Janeiro: Forense Universitária, 2012).

${ }^{19}$ G. Bachelard, J. A. Miller, G. Canguilhem e M. Foucault, "Epistemologia" ["Épistémologie"], Revue Tempo Brasileiro, 28, 1972.

${ }^{20}$ G. Bachelard, J. A. Miller, "Epistemologia”, Revue Tempo Brasileiro.

${ }^{21}$ Louis Althusser, "Apresentação do posfácio de Pierre Macherey" [“Présentation de le postface du Pierre Macherey”]. In: Georges Canguilhem, O normal e o patológico (Rio de Janeiro: Forense Universitária, 2015), 243-248.
} 
dans la santé - et dans la Médecine Préventive, en particulier - sans que ceux-ci aient besoin de récuser, comme irrémédiablement technocratique et oppresseur, l'instrumentalité des sciences médicales et sanitaires, constitutive de son identité comme domaine. Sur cela, Arouca nous dit ceci:

Devant la réalité politique latino-américaine, dans laquelle l'État a fréquemment assumé totalement ou partiellement le contrôle des actions de santé, à travers différents systèmes de prévoyance, amène, comme tendance à ce que la Médecine Préventive s'éloigne progressivement, de ses relations avec la société civile pour s'approcher de l'État dans sa dimension technocratique.

Cependant, dans le noyau de ces contradictions, il s'est développé de nouvelles conceptions sur le rôle de le Médecine Préventive dans la transformation de la théorie de la Médecine, à travers une pratique théorique spécifique qui parvient à délimiter l'idéologique dans son intérieur...Telle possibilité de pratique a été possible à travers la divulgation de travaux comme ceux de Canguilhem, Bachelard, Althusser et Balibar, Foucault et Alii, Fichant et Pecheux, Bernis, Labastida, Silmon, Piaget, Boltanski, Illich, Jantsche, Heckenhauser et d'autres, qui ont, dans un processus d'ouverture de nouveaux horizons dans les sciences sociales fourni de nouveaux instruments pour l'analyse de la médecine ${ }^{22}$.

On voit la forte présence de Canguilhem non seulement pour être le premier cité, mais aussi parce qu'une partie expressive des autres auteurs cités a été directement ou indirectement influencée par lui. Comme le remarque Foucault.

Mais ôtez Canguilhem et vous ne comprenez plus grand-chose à toute une série de discussions qui ont eu lieu chez les marxistes français; vous ne saisissez pas, non plus, ce qu'il y a de spécifique chez des sociologues comme Bourdieu, Castel, Passeron, et qui les marque si fortement dans le champ de la sociologie; vous manquez tout un aspect du travail théorique fait chez les psychanalystes et en particulier chez les lacaniens. Plus: dans tout le débat d'idées qui a précédé ou suivi le mouvement de 1968, il est facile de retrouver la place de ceux qui, de près ou de loin, avaient été formés par Canguilhem. ${ }^{23}$

En effet, l'histoire épistémologique de Canguilhem, en valorisant l'erreur, l'accident, l'obstacle comme partie de la construction des concepts scientifiques, permettait d'échapper de la camisole de force du déterminisme historique, d’esprit néo-hégélien, consolidant de nouvelles perspectives ouvertes dans le panorama philosophique par les lectures phénoménologiques et structuralistes que renouvelaient le marxisme. En outre, ses recherches sur les sciences de la vie montraient la direction en cheminant, en ouvrant des possibilités de se concevoir et d'administrer

\footnotetext{
${ }^{22} \mathrm{~S}$. Arouca, O dilema preventivista: contribuição para a compreensão e crítica da Medicina Preventiva [Le dilemme préventivist: contribution pour la comprébension et la critique de la médecine préventive] (São Paulo: Ed. UNESP/Rio de Janeiro: Ed. FIOCRUZ, 2003), 142 .

${ }^{23}$ M Foucault, "La vie: L’expérience et la science”, Revue de métaphysique et de morale, 90, 1(1985): 3-14, 3.
} 
avec l'inséparabilité entre histoire et science, en réalisant dans la pratique académique concrète une approche entre rationalisme et matérialisme extrêmement bienvenue pour la tradition marxiste. ${ }^{24}$

C'est toutefois, Lo normal y lo patologico 25 , édition argentine de l'œuvre alors inédite au Brésil qui serait en effet directement influente dans la construction du Dilemme préventiviste. Non seulement par la méthode utilisée par Arouca, plus apparentée avec l'archéologie du savoir de Foucault et le structuralisme marxiste de Althusser, ${ }^{26}$ mais parce que c'est là que se trouve ce qui me semble l'argument central et original du Dilemme et qui deviendra canonique dans le domaine de la Santé Collective: la question de la "normativité" comme clé de lecture des processus de santé-maladie. En recevant de Canguilhem la définition de la santé comme capacité de réponse aux infidélités du courant de la vie qui vont mettre à l'épreuve la valeur pratique de nos processus physiques et mentaux, s'ouvre un chemin pour, d'un côté, comprendre les valeurs qui étaient derrière un certain ensemble de propositions données comme promotrices ou récupératrices du "normal", ou non pathologique, comme un discours préventif. D'un autre côté, les insuffisances perçues dans les pratiques préventives rendaient possible de questionner l'efficacité normative de ces discours, ouvrant l'espace à la critique, à l'intérieur de la propre médecine préventive au milieu de ses tendances conservatrices. C'est ainsi qu'Arouca put identifier la stérile factorisation du social en critiquant le modèle de l'Histoire Naturelle de la Maladie, de Leawell et $\mathrm{Clark}^{27}$, mais en même temps, percevoir dans le modèle écologique d'explication causale ici proposée, des forces pour réclamer un abordage de la prévention qui ne soit pas restreint à une lecture strictement médicale. C'est ainsi qu'il perçoit également le mouvement de la médecine communautaire comme un changement normatif devant les limites de la prévention dans les domaines de la Médecine et de la Santé Publique traditionnelles et les dangers et les opportunités qui là s'établissent et que nous accompagnons jusqu'aujourd'hui dans le domaine de la Santé Colletive. Quand, par exemple, nous cherchons à affiner les relations entre le domaine institutionnel de la santé (formation de cadres, organisation de services, actions offertes) et les processus socio-politiques plus amples, ne sommes-nous pas en train d'ouvrir le domaine de la santé sur de nouveaux sujets et sur de nouvelles pratiques sociales, selon de nouveaux modèles normatifs, ou simplement en médicalisant le social en cachant derrière des normalisations scientifiques et techniquement conduites à l'imposition acritique d'un même modèle de réponses normatives?

La centralité de cet argument, et sa double face, de dénonciation du caractère conservateur et imperméables aux valeurs sociales émancipatrices de la Médecine et de la Santé Publique hégémoniques, d'un côté, et, de l'autre, de pari politique en alternatives reconstructives émergentes de l'intérieur de la propre structure de discours et de pratiques critiquées, marques de la Santé Collective, vont apparaittre joliment illustrées peu de temps après dans la défense de thèse de Arouca, faite en juillet 1976. En novembre de la même année fut lancé le premier numéro de la revue Santé en Débat, publiée par le CEBES - Centre Brésilien d'Études de la Santé, organisation dont l'importance dans la construction du domaine de la Santé Publique est comparable à celle de l'ABRASCO. Cette revue a divulgué et a catalysé intensément la production conceptuelle et politique du Mouvement de Réforme Sanitaire et s'est constitué ainsi comme le véhicule de sa construction. Dans son premier numéro, parmi d'autres contributions importantes, Santé en Débat a publié deux articles, séquentiel dans l'ordre dans lequel ils sont disposés dans la publication - et, en effet, ils constituent des contributions entrelacées - qui peuvent très bien être lues comme une proposition programmatique pour la Santé Collective. Sergio Arouca publie

\footnotetext{
${ }^{24}$ Dominique Lecourt, "La historia epistemológica de Georges Canguilhem" ["L’histoire épistémologique de Georges Canguilhem”]. In: G. Canguilhem, Lo normal y lo patológico (Buenos Aires: Siglo XXI, 1971), VII-XXX.; L. Althusser, "Apresentação do posfácio de Pierre Macherey."

${ }^{25}$ G. Canguilhem, Lo normal y lo patológico [Le normal et le pathologique] (Buenos Aires: Siglo XXI, 1971).

${ }^{26}$ E. D. Nunes, "Por uma arqueologia da Medicina Preventiva" ["Par une arquélogie de la Médecine Preventive"]. In: $O$ dilema preventivista: contribuição para a compreensão e crítica da Medicina Preventiva, org. Sergio Arouca (São Paulo: Ed. UNESP/Rio de Janeiro: Ed. FIOCRUZ, 2003), 103-108.

${ }^{27}$ H. R. Leavell \& E. G. Clark, Textbook of Preventive Medicine [Manuel de la Médecine preventive] (New York: MacGraw-Hill, 1953).
} 
L'Histoire Naturelle des maladies et Anamaria Tambellini Arouca, alors épouse de Sergio, signe avec lui le second article appelé Médecine de communauté: implications d'une théorie.

Dans le premier article 28 , en vérité un morceau de sa thèse (deuxième partie du quatrième chapitre), nous voyons Arouca déconstruire le modèle de l'Histoire Naturelle des Maladies (HNM) de Leavell et Clark. En remarquant dans l'ensemble de la thèse et dans l'examen des contradictions et des brèches propres au processus de construction de la Médecine Préventive, la rhétorique parait acquérir ici un ton bien plus dur (parce qu'unilatéral) dans la critique du caractère conservateur du modèle de HND. Ce qui est mis en évidence ici, c'est ce que je résumerais comme la "cécité normative" de la médecine. Le social apporté au modèle ne serait qu'un adjectif et comme tel, śajouterait à lui comme un "mythe", une apparente réforme de la médecine, mais qui en vérité laissait intouché son noyau substantif. Et cela se présenterait principalement par 1) Décomposer les aspects sociaux associés au modèle, les subordonner à l'explication des processus de la maladie comme conditions indépendantes entre elles, comme si elles ne faisaient pas partie d'une totalité qui les qualifie, intègre et hiérarchise selon des modes de production et de reproduction matérielle et culturellement déterminées, (un social naturalisé, donc). 2) Maintenir le modèle causaliste d'explication des phénomènes, répétant le modèle des sciences naturelles même s'il l'étend à de multiples facteurs utilisant le concept des réseaux causaux, le raisonnement linéaire et unidirectionnel des causes efficaces continuait, malgré tout intact. 3) Maintenir dans l'espace traditionnel des institutions de santé le processus de travail à être privilégiée dans la construction de réponses normatives aux exigences de la santé, centralisées dans la consultation médicale, maintenant les mêmes sujets et valeurs comme détenteurs du monopole des décisions normalisatrices sur la santé, la maladie et les soins.

Lu de forme isolée, cet article parait contradictoire avec le type d'analyse favorisée par la référence à l'œuvre de Canguilhem, dont Arouca profitera d'une façon si puissante dans sa thèse. Il y a comme un déterminisme, un destin immanément conservateur du cadre de la HNM, duquel disparaissent les multiples voix qui construisaient ce cadre conceptuel, parmi lesquelles celles qui n’étaient pas encore capables de transformer plus radicalement les savoirs de la Médecine et de la Santé Publique traditionnels. Celles-ci qui cherchaient dans le concept de prévention / préventisme des réponses à des problèmes qui, dans ce qu'ils ont de plus fondamental, étaient aussi une préoccupation du naissant mouvement de Réforme Sanitaire; comment intégrer médecine et santé publique, et comment incorporer au mode de connaitre et de faire des deux le caractère social des processus santé-maladie? Il semble disparaittre aussi du passage, hors du contexte de la thèse, la compréhension canguilhemienne du sens de normativité vitale qu'aucuns des savoirs sur la santé ne peuvent dispenser. C'est la compréhension canguilhemienne qui permettait de comprendre la tension qu'un cadre comme celui de la HND provoquait sur les formes traditionnelles de la Médecine et de la Santé Publique, favorisant les paris de rénovation de la Médecine de Communauté comme elle était alors appelée.

Cependant, dans l'article suivant, on passe de cette posture de simples dénonciations à une ouverture concrète aux possibilités d'une transformation effective des pratiques.

Pendant les dernières décennies, une série de concepts ont été présentés comme des solutions pour ladite crise du secteur de la santé, comme 'Médecine Préventive', 'Médecine Compréhensive' et, plus récemment 'Médecine de Communauté'. En réalité, de tels concepts, issus d'un mécanisme d'adjectivation de la médecine, ne possèdent pas derrière eux un cadre théorique qu'explique la réalité à laquelle ils s'appliquent... L'absence d'un cadre théorique qui rende compte de cette 'crise' génère des positions qui, en maintenant une distanciation théorique, se constituent

\footnotetext{
${ }^{28}$ S. Arouca, "História Natural das Doenças" [“Histoire naturelle des maladies”], Saúde em Debate, [vol.] 1, 1 (1976): 15-19.
} 
comme un ensemble désordonné d'expériences empiriques que ne posent pas de problèmes à la connaissance... Toutefois l'ensemble des expériences générées par ces oppositions peut être un grand stimulant de la production théorique. ${ }^{29}$

Et l'auteur qui est alors appelé à fournir des subsides pour construire les concepts nécessaires à la production de réponses aux nouvelles nécessités pratiques du domaine de la santé, n'est personne d'autre que Canguilhem:

Nous prétendons dans cet article, à partir des théories de Georges Canguilhem, vérifier quelques-unes des explications possibles en relation à cet ensemble de pratiques dénommées médecine de communauté. ${ }^{30}$

Il s'ensuit alors un compte rendu des contributions de Canguilhem. Même s'il n'y a pas de références bibliographiques dans l'article, le travail présenté est au fond, Le normal et le pathologique. Dans le compte rendu soigné, bien que synthétique, ce qui est mis en évidence par le couple Arouca, c'est la construction du concept de santé et de maladie menée à sa fin par Canguilhem à partir de son concept central de normativité vitale et sa relation avec les "allures de la vie". C'est à dire que la santé devient comprise comme une réponse satisfaisante, dans l'“expérience vécue”, de nos capacités de répondre aux "infidélités" qui menacent notre intégrité et notre bien-être, qualifiant ainsi santé et maladie comme des polarités valorisantes (positive ou négative) en relation à notre capacité de répondre (ou non) aux exigences normatives, et rendre ainsi possible la production de normes qui prennent en compte nos attentes et nécessités comme vivant. Dans cette même perspective, et en s'appuyant sur la critique de Canguilhem, les prétentions scientifiques de définir physiologiquement la maladie par la valeur négative des phénomènes pour le fonctionnement maximal de la totalité organique ("on ne dicte pas scientifiquement les normes à la vie"), suggèrent également la nécessité de reconstruire les relations entre les sciences et les techniques dans la médecine. Elles proposent que se sauvegarde la précédence pratique de ces dernières, ainsi comme quand on revoit les espaces et les ressources des pratiques de santé, de manière à rendre possible aux personnes de "s'approprier" leurs logiques et ressources pour leur propre bénéfice, au lieu d'être exproprier de leurs expériences et intérêts en santé par le "monde insolite" de ces ambiances et procédés technique. Et, ainsi même en remarquant que ce n'est qu'une parmi d'autres possibles contributions théoriques innovatrices autour de la médecine communautaire, les auteurs concluent

Ainsi, nous devons à partir de Canguilhem:

-Libérer la vie pour qu'elle questionne la science sur l'expérience, à travers l'expérience des sujets concrets,

-Libérer l'homme dans son 'expérience', ce qui signifie lui fournir les moyens pour comprendre et contrôler son 'allure de la vie 31

\footnotetext{
${ }^{29}$ A. T. Arouca e S. Arouca, "Medicina de comunidade: implicações de uma teoria," Saúde em Debate [vol.] 1, 1 (1976): 20 $23,20$.

${ }^{30}$ A. T. Arouca e S. Arouca, "Medicina de comunidade: implicações de uma teoria", 20.

${ }^{31}$ A. T. Arouca e S. Arouca, "Medicina de comunidade: implicações de uma teoria", 23.
} 


\section{Canguilhem et le Département de Médecine Préventive de la Faculté de Médecine de 1'Université de São Paulo.}

Vers le milieu des années 1970, persécutés politiquement, le groupe de professeurs et d'étudiants catalysés par Arouca à l'UNICAMP commence à se disperser. ${ }^{32}$ Ce groupe était composé de noms qui seront plus tard d'une grande importance pour la santé du pays, au-delà du propre couple Arouca, citons celui de David Capistrano Costa Filho (1948-2000) e Eleutério Rodriguez Neto (1946-2013). Arouca est transféré en 1976 à l’École Nationale de Santé Publique. Le Département de Médecine Préventive et son programme de post-graduation, qui développait aussi le rôle d'un important centre d'attraction et d'irradiation de la pensée critique de la naissante Santé Collective, a reçu aussi des personnes qui en faisaient partie et des sympathisants de ce groupe, par exemple Eleutério Rodriguez Neto, Ricardo Lafetá Novaes (1945-1998) et Hillegonda Maria Dutilh Novaes. Là, se rencontraient déjà, Cecilia Donnangelo et Guilherme Rodrigues da Silva (1928-2006), deux des plus grands leaders politiques et intellectuels de la construction de ce champs de recherche. Dans cette ambiance, la forte influence de Canguilhem va également se faire remarquer.

Ricardo Lafetá Novaes a été un de ceux qui, en étroite relation avec Arouca et son groupe, et partageant avec ceux-ci l'intérêt de réfléchir de façon critique sur les concepts qui étaient la base des pratiques de santé, a développé dans la Médecine Préventive de l'USP, sous l'orientation du Prof. Guilherme Rodriguez da Silva, une maitrise où l'influence de Canguilhem est marquante. La Santé et les concepts ${ }^{33}$ est contemporaine des travaux de Arouca, cités plus haut, et lui ressemblent. Des références méthodologiques centrées sur Althusser, jusqu'à la défense de la nécessité de reconstruction conceptuelle pour rendre effectifs les changements signalés par les propositions de réforme de l'attention et de l'enseignement médical, mais spécialement par le soutien à l'épistémologie historique de Canguilhem et au potentiel reconstructif de son concept de normativité en santé, cette étude de Novaes résonne comme une continuité de l'investissement théorique proposé dans l'article de la revue Santé en Débat. ${ }^{34}$

Maria Novaes propose une continuité du dialogue avec Canguilhem mais d'une manière différente de Lafetá, elle s'intéresse plus à l'historien des sciences qu'au philosophe - quoique par la force du propre référentiel de l'épistémologie historique, sa récupération de l'histoire de la puériculture ${ }^{35}$ a également été guidée par la réflexion sur les bases épistémologiques des sciences médicales, les discussions sur cette connaissance s'appuyaient en même temps sur les informations historiques qu'elle avait recueillies. Dans son étude, le recours à Canguilhem, quoiqu'il s'appuie également sur la question de la normativité, qui, comme nous l'avons vu, a été le pont qui a relié les territoires de la Santé Collective à l'œuvre de Canguilhem, a été fondamentale pour caractériser la particularité de l'objet de l'histoire des sciences médicales. Pour cela, au-delà du Le normal et le pathologique, il a eu recours au texte classique L'objet de l'histoire des sciences, ${ }^{36}$ et à $1^{\prime}$ Idéologie et rationalité, dans sa version française originale. ${ }^{37}$

Du travail que Cecília Donnangelo était en train de développer dans le Département résulte également un gain important aux contributions de l'épistémologie historique en Santé Collective qui m'intéresse d'examiner ici en particulier. Vu qu'il a produit une inflexion assez

\footnotetext{
${ }^{32}$ Comissão da Verdade e Memória Otávio Ianni (Relatório Final, Unicamp: Universidade Estadual de Campinas, 2015) [Commision de la Vérité et de la Mémoire Otávio Ianni (Rapport final, Unicamp: Université d'état de Campinas].

33 R. L. Novaes, $A$ saúde e os conceitos (Dissertação de mestrado).

${ }^{34}$ A. T. Arouca e S. Arouca, "Medicina de Comunidade: implicações de uma teoria".

${ }^{35}$ H. M. D. Novaes, A puericultura em questão.

${ }^{36}$ G. Bachelard, J. A. Miller, "Epistemologia", Revue Tempo Brasileiro

${ }_{37}$ G. Canguilhem. Idéologie et rationalité dans l'bistoire des sciences de la vie: Nouvelles études d'histoire et de philosophie des sciences (Paris: Vrin, 1977)
} 
original et qu'il a résulté en une ligne de recherche en cours jusqu'aujourd'hui dans le Département et également influente en dehors de lui.

Dans Santé et société ${ }^{38}$, considérée avec Le dilemme préventiviste, comme œuvre fondatrice de la Santé Collective, comme nous l'avons déjà signalé, nous trouvons Canguilhem dès les premiers paragraphes. Et ici, comme dans les travaux cités plus haut, le recours au philosophe se doit à sa conception de la normativité de la vie et à la dislocation qu'il impose aux conceptions traditionnelles de la santé et de la maladie - et ses conséquences sur les façons de penser les relations entre physiologie et pathologie et spécialement entre science, technique et pratique.

La clinique est inséparable de la thérapie et celle-ci est une technique $\mathrm{d}$ 'instauration et de restauration du normal dont l'objectif, de savoir que la satisfaction subjective d'une norme qui est instaurée, échappe à la juridiction du savoir objectif. On ne dicte pas des normes scientifiques à la vie (...) La propre vie (...) est qui introduit dans la conscience humaine les catégories de la santé et de l'infirmité. Ces catégories sont biologiquement techniques et subjectives et non pas scientifiques et objectives. ${ }^{39}$

Donnangelo poursuit en dialoguant avec Le normal et le pathologique tout au long du premier chapitre, Médecine: pratique technique - pratique sociale, de la première partie du travail dénommée Médecine et structure sociale. Cette œuvre est fondamentale pour la construction de ce qui deviendrait plus tard la marque principale de la contribution de l'auteur dans le domaine de la Santé Collective: la nécessité d'explorer la "consubstantialité technico-social des savoirs et des pratiques de santé”. Ce fut, sur cette voie que, encore une fois, Canguilhem a été appelé à soutenir une direction programmatique de la construction des pratiques en santé alors recherché par le mouvement sanitaire brésilien - et motivée également ici par la crise de la médecine et par la critique des déficiences des modèles de réforme proposés par le préventivisme et par la Médecine de Communauté, ou Médecine Communautaire, comme l'appelle Donnangelo, s'alignant sur celles déjà réalisées par Arouca ${ }^{40}$ et Guilherme Rodrigues da Silva ${ }^{41}$. En mettant en évidence la priorité ontologique du pathologique sur le physiologique, celui-ci compris comme un obstacle expérimenté par une "allures de la vie" donnée, réclamant de nouvelles normes de régulation qui devraient être produites pour le corps ou par son milieu, Canguilhem dit clairement que les lectures sur les corps et les milieux effectuées par les sciences de la vie et de la santé, selon les intérêts normatifs qui produiront des techniques d'interventions normalisatrices, ces lectures ne sont pas les expressions objectives immanentes d'une nature dévoilée par la connaissance méthodique des sciences, mais avant tout, elles révèlent la précédence et l'ascendance des "valeurs" sur l'objectivité scientifique et la relation de ces valeurs avec les nécessités et les possibilités inscrites dans la concrétude des expériences d'être vivant.

Cette construction, comme nous l'avons déjà signalé, permettait d'établir un dialogue réussi avec les positions du matérialisme historique, si importante dans le milieu académique de la Santé Collective. Elle fournissait des éléments qui ne donnaient pas seulement une densité conceptuelle à la dénonciation du mythe de la neutralité axiologique des sciences et des techniques, mais elle créait aussi la possibilité d'un dialogue théorico-méthodologique entre l'investigation des aspects socio-politiques, en relation avec les expériences concrètes de la santé, de la maladie et des soins et le plan épistémologique des savoirs et des pratiques de la médecine.

Ainsi, en dialoguant avec différents intérêts et auteurs, dans le marxisme et hors de lui (on remarque là l'importance de Foucault), provenaient diverses manières de profiter de l'œuvre de

\footnotetext{
${ }^{38}$ M. C. F. Donnangelo e L. Pereira, Saúde e sociedade [Santé et société] (São Paulo: Duas Cidades, 1976).

${ }^{39}$ M. C. F. Donnangelo e L. Pereira, Saúde e sociedade, 16-17.

${ }^{40} \mathrm{~S}$. Arouca, O dilema preventivista: contribuição para a compreensão e crítica da Medicina Preventiva.

41 G. R. Silva, "Origens da Medicina Preventiva como disciplina do ensino médico" ["Origines de la Médecine Préventive comme discipline de l'enseignement médical”], Rev. Hosp. Clin. Fac. Med. S. Paulo, 28 (1973): 91-96.
} 
Canguilhem dans le Département de Médecine Préventive. En dialoguant spécialement avec Althusser, par exemple, Novaes a mis en évidence spécialement le composant idéologique et la dénonciation de la non-transparence du sens normatif des pratiques d'exercice et d'enseignement de la médecine, par la force des mécanismes structuraux de domination de classe.

Nous pensons que la santé est un processus organique surdéterminé par des normes vitales supérieures qui permettent à l'organisme d'être normatif, processus qui est une nouvelle fois surdéterminé puisque la VALEUR dominante est décurrente des représentations et des exigences de la structure sociale qui, étant données les relations de production, institue les classes et leur lutte. La Santé et la maladie sont ainsi beaucoup plus des valeurs sociales historiquement mises en place que la simple expression de la situation biologique de l'organisme (en général) dans un milieu donné. ${ }^{42}$

Donnangelo, en dialogue également avec des auteurs structuralistes, mais cherchant spécialement chez Gramsci des opportunités pour penser les aspects structurés et structurants des pratiques en santé dans les formations sociales en termes de conflit et de contradictions entre projets (et sujets) sociaux (hégémoniques et contre hégémoniques), focalisera ses analyses beaucoup plus sur l'action politique que sur l'aspect de la domination idéologique à proprement parlé. Dans la première, et la plus importante partie de son travail, l'auteur trace un panorama de la confrontation des pratiques en santé dans les formations sociales capitalistes, synthétisée dans l'extrait ci-dessous:

La redéfinition de la médecine comme pratique sociale apparaît de manière marquée au XVIII'̀me siècle, à travers son extension institutionnalisée sur toute l'étendue de la société, imprégnant le processus politique et économique d'une forme particulière. Ce ne sont pas les soins médicaux qui se généralisent alors mais ce que l'on pourrait considérer de manière approchée, comme une extension du domaine de normativité de la médecine à travers la définition des nouveaux principes référents au signifié de la santé et de l'interférence médicale dans l'organisation de la population et de ses conditions générales de vie. ${ }^{43}$

Cette médicalisation de la vie sociale devient alors la base de son analyse critique sur les relations entre la santé et la société et la consubstantialité technico-sociale des pratiques de santé. Mais son analyse de la médicalisation dans les moules manipulés par la médecine communautaire va signaler non plus le sens de "contrôle" de la force de travail, comme dans les premiers temps du processus de transformation capitaliste des sociétés occidentales, mais l'extension (à faible coût) de la consommation des services de santé, consolidant la pratique médicale comme travail social et légitimant politiquement le modèle normatif (non transparent) réitéré par les sciences et les techniques de la médecine.

Avec cela, Santé et société ne produit pas seulement une charpente théorique pour l'analyse de la Médecine Communautaire, mais construit des balisages conceptuels pour une sociologie de la santé de manière plus générale. ${ }^{44}$ Et, quoiqu’elle extrapole les possibilités de traitement dans

\footnotetext{
42 R. L. Novaes, "A saúde e os conceitos", 176.

${ }^{43}$ M. C. F. Donnangelo e L. Pereira, Saúde e sociedade, 47.

${ }^{44}$ L. Pereira, "Capitalismo e saúde" ["Capitalisme et santé"]. In: Saúde e sociedade, orgs. M. C. F. Donnangelo e L. Pereira (São Paulo: Duas Cidades, 1976), 95-124.
} 
l'espace de cet article, il ne sera pas difficile d'imaginer la richesse des possibilités qui se sont alors ouvertes à la pensée critique dans le domaine de la Santé Collective, par la combinaison de l'ouverture à la consubstantialité technico-social des pratiques en santé et la possibilité de mener son investigation à l'intimité de la conformation des sciences et des techniques sur lesquelles ses pratiques sont fondées, par le dialogue productif établit avec l'épistémologie historique. L'étude la plus significative qu'elle a traité, pour le moins celle qui s'est déployée dans la production des interlocuteurs les plus proches de Cécilia, va se pencher sur la question de la médecine comme "travail", entendu non pas dans la stricte condition d'une activité productive "infrastructurelle" des sociétés capitalistes, mais comme pratique de sujets (les médecins) liés de façons contradictoires (comme dominés et dominants) à la manœuvre technoscientifique de normativité posées pour le contrôle de la santé dans le contexte des modes de reproduction sociales historiquement constitués. ${ }^{45}$ Nous ne commenterons pas toute cette extensive et riche production dans son ensemble. Je vais me concentrer seulement sur le côté théorique sur lequel s'est fortement resserré le dialogue avec l'épistémologie historique.

\section{Canguilhem et la Théorie du Processus de Travail en Santé}

Ricardo Bruno Mendes Gonçalves (1946-1996), le plus proche collaborateur de Cecilia Donnangelo, a défendu sa maîtrise, Médecine et histoire: Racines sociales du travail médical peu de temps après l'habilitation de Donnangelo. Son travail est tellement influencé par le sien qu'il semble presque le compléter, dans le sens discerné par Luiz Pereira (1933-1985) dans l'argumentation à l'auteur: "Justement, parce que l'auteur a appris les déterminations infrastructurelles de la pratique médical par les fonctions de ces dernières, elle laisse une lacune dans sa thèse dans ce qui se réfère à de telles déterminations: oublie de penser la pratique médicale comme un travail". ${ }^{46}$

Ce fut, comme nous l'avons dit plus haut, le chemin qu'a suivi Ricardo Bruno dans sa maitrise. Il n'y a pas lieu ici d'explorer en détail les contenus de cette œuvre importante, je vais seulement distinguer brièvement, comment, en concevant ce qui serait plus tard sa Théorie du Processus de Travail en Santé (TPTS), elle a permis de poursuivre le dialogue de Canguilhem avec la Santé Collective. Dans ce sens, il est important de remarquer que dans la perspective conceptuelle de Médecine et histoire, le matérialisme et l'histoire sont devenus non seulement une façon d'exprimer une prise de position politique, de dénonciation critique des directions conservatrices des pratiques de santé, mais a configuré positivement des possibilités de recherche théorique et appliquée d'un caractère que nous pouvons appeler de reconstructeur. Peut-être sous l'influence gramscienne, Ricardo Bruno s'est montré spécialement sensible au devenir historique et au rôle des sujets et de leurs interactions, permettant de tenter les possibilités reconstructives de l'intérieur même des pratiques de santé. Cette perspective est ouverte, premièrement, par la caractérisation dans Médecine et histoire de la double position des médecins dans les sociétés capitalistes: d'un côté, membres d'une élite intellectuelle qui formule les projets sociaux hégémoniques, basés sur la légitimité et le pouvoir pratique des sciences médicales, d'un autre travailleurs qui produisent des services et, comme tel, sont dominés, non seulement par les

\footnotetext{
${ }^{45}$ R. B. Mendes-Gonçalves, Medicina e história: raízes sociais do trabalho médico [Médecine et histoire: Racines sociales du travail médical] (Dissertação de mestrado, Universidade de São Paulo, 1979); R. B. Mendes-Gonçalves, Tecnologia e organiżação social das práticas de saúde: características tecnológicas so processo de trabalho em saúde na rede de centros de saúde do estado de São Paulo [Technologie et organisation sociale des pratiques en santé: caractéristiques technologiques du processus de travail dans le réseau public de centres de santé de l'état de São Paulo] (Tese de doutorado, Universidade de São Paulo, 1986); L. B. Schraiber, Contribuição ao estudo da educação [Contribution à l'étude de l'éducation médicale] (Dissertação de mestrado, Universidade de São Paulo,1980); L. B. Schraiber, Medicina liberal e incorporacãa de tecnologias: as transformações históricas da autonomia profissional dos médicos em São Paulo [Médecine libérale et incorporation de Tecnologies: les transformations historiques dans l'autonomie professionelle des médecins á São Paulo] (Tese de doutorado, Universidade de São Paulo, 1989).

${ }^{46}$ M. C. F. Donnangelo e L. Pereira, Saúde e sociedade, 111.
} 
relations de production qui progressivement définissent les modes de travail et de vie, mais aussi par le pouvoir progressif de la technologie pour déterminer le sens et la valeur de sa connaissance et pour déterminer sa place dans les processus (parcellaires) du travail en santé. C'est justement cette insertion contradictoire qui rend possible, selon Ricardo Bruno, la perception des possibilités émancipatrices d'une pratique théorique que se voue à l'exploration des relations entre les technologies et les sciences de la santé et ses sujets. Parier sur l'investigation empirique et la réflexion critique autour des technologies du travail médical (y compris sa relation avec les sciences) peut selon cet auteur produire des tensions et transformer les positions des sujets, les relations de pouvoir et ainsi, les valeurs et les normativités qui orientent nos pratiques en santé. ${ }^{47}$

Un des résultats de ce dialogue fertile entre la compréhension de la médecine comme travail et l'épistémologie historique de Canguilhem, commencé avec Médecine et histoire, va apparaittre avec toute sa force dans un travail originalement publié en 1985 et réédité en 1990,48 et qui eut un grand impact dans la Santé Collective. Dans cette étude, c'est la relation entre la science épidémiologique et la pratique médicale qui est examinée, développant la thèse que, dans le travail concrètement opéré dans le contexte du développement historique de la clinique moderne, au long des XIX $^{\text {ème }}$ et XX $^{\text {ème }}$ siècle, les bases normatives de l'epidemiologie sont subsumées dans la pratique médicale à la perspective clinique. Le Canguilhem du Le normal et le pathologique est central dans la construction du référentiel théorique du texte (pages 41-53), principalement pour comprendre comment la normativité hygiéniste à l'origine de l'épidémiologie, qui ambitionnait le maniement des déterminants sociaux des processus de maladie, a été subsumée comme travail concrètement opéré dans la médecine au maniement individualisé de ce social, il est exprimé comme-tel dans le modèle d'Histoire Naturelle de la Maladie.

Dans son doctorat, ${ }^{49}$ Ricardo a continué son entreprise théorique en densifiant et en mûrissant son cadre conceptuel et en l'appliquant, alors, à une recherche empirique autour des processus de travail concrètement opérés dans les Centres de Santé du réseau de São Paulo. La dette de cette entreprise avec l'architecture conceptuelle que nous venons de discuter est explicite dans les références bibliographiques et semble synthétisée dans les extraits ci-dessous:

(La pratique médicale) est un travail, enfin, parce qu'elle vise l'obtention d'effets déterminés, elle cherche à altérer un état des choses établi comme manque, et en faisant cela, s'aide des conceptions scientifiquement élaborées sur le corps pour traiter avec quelque chose qui échappe aux limites du corps, encore que cela puisse être appris ainsi: le pathologique, comme expression d'une normalité spécifique. (...) ... Les analyses de Canguilhem et Donnangelo permettent de comprendre... que le corps anatomophysiologique ne se définit plus comme normal ou pathologique s'il ne prend pas comme référence ces structures de normativité extra-biologiques et qui, en conséquence, la normativité est immédiatement contenue dans l'objet du travail du médecin. ${ }^{50}$

Comprises comme un travail, il soutient que les pratiques médicales admettent d'être analysées comme un ensemble de connaissances de type scientifique ("savoir que"), savoirs technico-opératoire ("savoir comment") et une correspondante instrumentalité matérielle, tous intégrés et qualifiés par des normativités relatives à des modes déterminés des "allures de la vie" et

\footnotetext{
47 J. R. C. M. Ayres, "Ricardo Bruno: história, processos sociais e práticas de saúde" ["Ricardo Bruno: l'histoire, les processus sociaux et les pratiques de santé”], Ciência e Saúde Coletiva, [vol.] 20, 3 (2015): 905-912.

48 J. R. C. M. Ayres, "Reflexão sobre a articulação entre a investigação epidemiológica e a prática médica, a propósito das doenças crônicas e degenerativas" ["Réflexion sur la relation entre la recherche épidémiologique et la pratique médicale, en ce qui concerne les maladies chroniques et degeneratives"]. In: Epidemiologia: teoria e objeto [Epidémiologie et object], org. D. C. Costa (São Paulo: Hucitec/Rio de Janeiro: Abrasco, 1990), 39-86.

${ }^{49}$ R. B. Mendes-Gonçalves, "Tecnologia e organização das práticas de saúde”.

${ }^{50}$ R. B. Mendes-Gonçalves, "Tecnologia e organização das práticas de saúde”, 66-68.
} 
configurée comme telle (travail en santé) seulement dans les formes concrètes de son opération. En conséquence, toute déconstruction critique des pratiques en santé trouvera un puissant recours dans l'appréhension des sens effectivement assumés par ses sciences dans la manière de comment ils s'articulent avec le travail concrètement opéré dans son domaine de normativité. Dans la même direction, mais en sens inverse, nous pouvons assumer que la reconstruction des pratiques en santé dépend de notre réussite dans la création de conditions politiques pour que de nouveaux horizons de normativité puissent être socialement reconnus et validés et (étant donné que) la valeur des sciences (et des techniques) qui les peut faire valoir soit concrètement expérimentée.

En raison de la fragilisation de sa santé et de sa mort précoce, Ricardo Bruno n'a pas eu l'opportunité de donner de plus amples développements théoriques à ce cadre, excepté dans quelques productions plus ponctuelles, comme sa discussion sur les nécessités de santé ${ }^{51}$ et des commentaires à partir des travaux de ses élèves ${ }^{52}$. Néanmoins, Lilia Schraiber, Maria Ines Baptistella Nemes et d'autres collaborateurs et élèves, nombreux et de diverses régions du pays, ont donné suite à des programmes de recherche dérivés des TPTS ou en dialogue avec cette production - à commencer par la propre expérimentation de nouveaux agencements technologiques pour les pratiques de l'attention primaire à la santé dans une unité de base, le Centre de Santé École Samuel B. Pessoa. ${ }^{53}$

Dans plusieurs de ces productions, la pensée de Canguilhem continue d'être, directement ou indirectement, une référence importante qui indique, dans ses succès et dans ses omissions, les exigences de rénovation critique auquel un travail intellectuel vigoureux et rigoureux comme le sien incite toujours.

\section{Canguilhem et la Santé Collective aujourd'hui, quelques refléxions finales}

La diffusion de l'œuvre de Canguilhem s'est amplifiée dans la Santé Collective (et doit s'amplifier encore avec la récente publication de nouvelles œuvres en portugais), à tel point qu'il serait difficile d'évaluer les modes de son utilisation dans la production actuelle de ce domaine sans un laborieux inventaire, ce qui n'est pas le sujet ici. Quelques publications des dernières vingt années, dans des revues scientifiques, nous donnent cependant quelques indices sur la continuité du dialogue avec ce penseur dans divers centres académiques du pays et même à l'étranger ${ }^{54}$. Il est

51 R. B. Mendes-Gonçalves, "Práticas de saúde: processos de trabalho" ["Pratiques de santé: processus de travail e besoins"], Cadernos CEFOR - Textos 1 (1992)

${ }_{52}$ R. B. Mendes-Gonçalves, Prefácio a J. R. C. M. Ayres [Préface], Epidemiologia e emancipação [Epidémiologie et emancipatrice] (São Paulo: Hucitec, 1995), 13-20 e R. B. Mendes-Gonçalves, "Seres humanos e práticas de saúde: comentários sobre 'razão e planejamento"' ["Les humains et les pratiques de santé: Commentaires sur 'Raison et planification"']. In: Razãa e planejamento: reflexões sobre política, estratégia e liberdade [Raison et planification: réflexions sur la politique, stratégie et liberte], org. E. Gallo (São Paulo: Hucitec/Rio de Janeiro: Abrasco, 1995), 13-31.

53 L. B. Schraiber (org.), Programação em saúde hoje [Programmation en santé aujourd'bui] (São Paulo: Hucitec, 1990) e L. B. Schraiber, M. I. B. Nemes, R. B. Mendes-Gonçalves (orgs.), Saúde do adulto: ações e programas na unidade [Santé de l'adulte: actions et programmes dans l'unité de base] (São Paulo: Hucitec, 1996).

54 A. Martins, "Novos paradigmas e saúde" ["Nouveaux paradigmes et santé"], Physis: Revista de Saúde Coletiva, [vol.] 9,1 (1999): 83-112; M. T. A. D Coelho e N. Almeida Fo., "Conceitos de saúde em discursos contemporâneos de referência científica" ["Concepts de santé dans des discours contemporrains de référence scientifique'], História, Ciências, Saúde Manguinhos, [vol.] 9, 2 (2002): 315-33; J. C. R. S. Soares e K. R. de Camargo Jr, "A autonomia do paciente no processo terapêutico como valor para a saúde" ["L'autonomie du patient dans le processos thérapeutique comme valeur pour la santé"], Interface - Comunic., Saúde, Educ., [vol.] 11, 21 (2007): 65-78; J. R. C. M. Ayres, "Uma concepção hermenêutica de saúde ["Une conception herméneutique de la santé"], Physis: Revista de Saúde Coletiva, [vol.] 17, 1 (2007): 43-62; K. R. de Camargo Jr., "As armadilhas da concepção positiva de saúde” [“Les pièges de la conception positive de la santé"], Physis: Revista de Saúde Coletiva, [vol.] 17, 1 (2007), 63-76; R. F. Puttini e A. Pereira Jr., "Além do mecanicismo e do vitalismo: a 'normatividade da vida' em Georges Canguilhem" ["Au-dèla du mécanisme et vitalisme: la 'normativité de la vie' dans Georges Canguilhem”], Physis: Revista de Saúde Coletiva, [vol.] 17, 3 (2007): 451-464; C. Resende, "O que pode um corpo? O método Angel Vianna de conscientização do movimento como um instrumento terapêutico" [“Qu'est-ce que peut un corps? La méthode Angel Viana de conscientisation du mouvement comme instrument thérapeutique"], Physis: Revista de Saúde Coletiva, [vol.] 18, 3 (2008): 563-574 ; D. Czeresnia, "Canguilhem e o caráter filosófico das ciências da vida" 
également difficile de juger, devant cette dispersion et augmentation de la production dans le domaine de la Santé Collective, si le dialogue avec cette œuvre continue a exercé le même effet d'enracinement dans l'expérience vécue, par l'intermédiaire de la réflexion sur la question de la normativité, les réflexions sur les sciences de la santé et les technologies en relation avec elles. Ce qui semble inexistant aujourd hui, c'est un recours à Canguilhem comme base conceptuelle d'une plate-forme politico-scientifique de nature programmatique pour le domaine de la Santé Collective comme un tout, comme dans les années 1970 (mas en existe-t-il une?). C'est dans ce sens, peutêtre, qu il serait nécessaire de réaliser des recherches plus régionales, non seulement dans le sens géographique, mais spécialement dans le sens de régions d'application de la rationalité de Santé Collective, pour évaluer la pénétration et la fécondité de ce dialogue. Serait-ce alors son œuvre qui agit effectivement dans la construction de concepts dans la Santé Collective?

Je vais chercher à répondre à cette question à partir de la réflexion sur quelques déploiements des contributions de Canguilhem autour de la Théorie du Processus de Travail en Santé dans ma propre production. Un tel examen qui sera fait ici, ne veut prétendre à aucun type de représentativité ample, mais pourra être utile pour signaler quelques possibilités et défis de la continuité de ce dialogue productif avec le penseur de la normativité vitale.

Partant de la consubstantialité technico-sociale des pratiques en santé (Donnangelo) et de la relation entre connaissance et processus de travail dans la compréhension de l'historicité et la configuration épistémologique des sciences de la santé (Mendes Gonçalves), j'ai développé deux lignes de productions académiques dans lesquelles je vois clairement les contributions de Canguilhem. Dans un premier temps, dans la recherche de la compréhension de la genèse et des fondements épistémologiques de l'épidemiologie, savoir stratégique dans l'opération de la normativité socio-sanitaire dans les processus de travail en santé. Dans un second temps, dans la critique du paroxysme techniciste des pratiques d'attention à la santé.

La propre manière par laquelle j’ai dénommé la première ligne de recherche, explicite l'influence de Canguilhem: critique "historico-épistémologique" de l'épidemiologie, travail de maitrise développé entre 1986 et 1991.55 En effet, si j’ai été incité par Ricardo Bruno à étudier cette science si importante pour le projet de Santé Collective à partir de la perspective des processus de travail en santé (ici, dans ses relations avec les pratiques de santé publique), ce fut avec la lecture de Canguilhem que j'ai rencontré un chemin cohérent pour le faire. Ceci parce que, pour autant qu'une science soit impliquée dans des processus concrets de travail, il y a des différences importantes, bien sûr, entre la manière par laquelle elle se laisse apprendre comme partie d'une technologie et les processus qui caractérisent le moment de sa production comme connaissance. Les intérêts et les compromis "internes" et "externes" de la communauté de scientifiques qui produisent l'épidemiologie, la matérialité technique qui promouvait ou détenait ses chemins, le degré et le type de développement de la rationalité qu'elle appliquait à ses problèmes d'investigation, et le contexte sociopolitique plus ample, qui sanctionnait ou non ses constructions, tout cela réclamait un référentiel qui apportasse à l'étude la perspective, non seulement d'une philosophie des sciences, une épistémologie, mais aussi, et avec elle, une sociologie des sciences.

Parmi les classiques qui déjà soutenaient d'une certaine façon ces perspectives de dialogue, Tomas $\mathrm{Kuhn}^{56}$ semblait clarifiant avec son concept de paradigme. "Structures

[“Canguilhem et le caractere philosophique des sciences de la vie”], Physis: Revista de Saúde Coletiva, [vol.] 20, 3 (2010): 709 727; S. Caponi, "Georges Canguilhem: del cuerpo subjetivo a la localización cerebral” ["Georges Canguilhem : du corps subjectif á la localisation cérébrale"], Salud Colectiva, [vol.] 6, 2 (2010): 149-161; A. Ostachuck, "La vida como actividad normativa y auto-realización: debate en torno al concepto de normatividad biológica en Goldstein y Canguilhem" ["La vie comme activité normative et l'auto-réalisation: débat autour du concept de normativité biologique chez Goldstein et Canguilhem”], História, Ciências, Saúde - Manguinhos, [vol.] 22, 4 (2015): 1199-1214.

${ }^{55}$ J. R. C. M. Ayres, Epidemiologia e emancipação (São Paulo: Hucitec/Rio de Janeiro: Abrasco, 1995).

${ }_{56}$ T. Kuhn, A estrutura das revoluções científicas [La structure des révolutions scientifiques] (São Paulo: Perspectiva, 1989). 
paradigmatiques", la manière par laquelle j'ai incorporé les contributions de cet auteur à mon propre cadre conceptuel, permettraient d'explorer des rationalités, des intuitions et des recours qui, de l'intérieur de la communauté scientifique de la santé, ont construit la science épidemiologique qui dialoguait (ou non) avec les exigences socio-sanitaires qui se constituaient comme un travail social au cours des différents moments historiques. Toutefois, Canguilhem nous enseigne que les sciences de la santé étaient, beaucoup plus immédiatement que les autres sciences, la propre vie humaine en établissant pour elle-même et son milieu les modèles normatifs capables de satisfaire ses nécessités et d'expérimenter ses possibilités. Il fallait alors une "hybridation" du cadre kuhnien avec une réflexion autour du problème de la "valeur" socio-vitale qui clarifiait la construction des concepts - ce point de départ de l'histoire épistémologique de Canguilhem. Ce fut ainsi que je suis parvenu à la formulation des "structures normative-paradigmatiques" pour entreprendre mon étude sur l'épistémologie de l'épidemiologie - que j'ai nommée "critique" historico-épistémologique pour signaler une valeur normative que je poursuivais moi-même dans mon étude, fondamentalement l'intérêt reconstructif du domaine de la Santé Collective.

Si le dialogue avec le concept de paradigme a cherché à suppléer un certain "déficit sociologique", fréquemment montré comme la limite de la méthode de Canguilhem ${ }^{57}$, de la même manière que, d'un côté, Canguilhem a permis d'amener pour l'analyse la question du sens normatif des sciences de la santé, ce dont manquait le cadre kuhnien, il y avait encore un autre défi à affronter dans la critique historico-épistémologique. Il concerne les difficultés de l'analyse des structures de rationalité pour rendre compte du "sens de l'action politique" qui les construit et reconstruit dans la poursuite des exigences normatives auxquelles elles répondent. Pour cela, on a eu recours dans ce travail ${ }^{58}$ aux notions de "projet anthropo-émancipateur et au projet technoconservateur" (tributaire de la tradition phénoménologique-existentialiste), avec un succès relatif dans ce contexte précis, ${ }^{59}$ mais la question du sens de l'action se posera toujours comme un défi pour l'épistémologie historique dans la mesure que ce ne sont pas seulement les domaines d'application des sciences qui se transforment en permanence, mais aussi leurs propres intérêts normatifs.

Cette ligne d'investigation a avancé en se focalisant d'une manière plus approfondie un des concepts développés par l'épidemiologie, justement celui qui a permis que son discours dépasse le seuil de la formalisation, ${ }^{60}$ śalignant entre les sciences hard de la santé au XX ${ }^{\text {ème }}$ siècle: le concept de risque. ${ }^{61}$ Dans cette étude, Canguilhem n'est pas utilisé aussi activement comme il l'avait été dans l'étude antérieure pour soutenir la construction théorico-méthodologique, mais le fait que ses contributions dans les sciences de la vie et de la santé ne soient pas évoquées tout au long du travail écarte l'hypothèse de que cela soit dû à quelques types d'oubli ou d'abandon. Comme il est probable que cela se passe dans d'autres productions dans le domaine de la Santé Collective, la contribution fondamentale de son épistémologie historique résulte presque comme un présupposé, un point de départ tacite - ce qui, même si cela semble une bonne explication, ne peut servir de justificatif pour la négligence envers la reprise critique d'un cadre si riche et si important pour la Santé Collective.

Dans une certaine mesure, je pense que ce fut le cas dans Sur le risque. Ici Canguilhem ne fait pas partie centralement de l'exposition de l'architectonique méthodologique de l'étude, même s'il est utilisé pour soutenir quelques-uns de ces traits fondamentaux, en particulier en ce qui concerne le sens récurrent de l'investigation à partir du concept depuis sa forme la plus aboutie (pour lui apprendre la rationalité, non pas l'immanence logique), et les concepts d'origine bachelardienne, de l'obstacle et de la rupture épistémologique. Le recours le plus important au

\footnotetext{
${ }^{57}$ M. T. A. D Coelho e N. Almeida Fo., "Conceitos de saúde em discursos contemporâneos de referência científica”.

${ }^{58}$ J. R. C. M. Ayres, Epidemiologia e emancipação [Epidémiologie et émancipation].

${ }^{59}$ R. B. Mendes-Gonçalves, Prefácio a J. R. C. M. Ayres, Epidemiologia e emancipação.

${ }^{60}$ M. Foucault, A arqueologia do saber [L'Arquéologie du savoir] (Rio de Janeiro: Forense-Universitária, 1987).

${ }^{61}$ J. R. C. M. Ayres, Sobre o risco: para compreender a epidemiologia [Sur le risque: pour comprendre l'épidemiologie] (São Paulo: Hucitec, 1997).
} 
long du texte se réfère à quelques-unes de ses discussions les plus acérée sur les différences et les proximités entre une idéologie scientifique et une science pleinement établie ${ }^{62}$, le concept de "milieu" et son importance pour les sciences de la santé, ${ }^{63}$ la lecture physiologique de la pathologie et son dogme de la continuité qualitative entre santé et maladie ${ }^{64}$ et la question plus contemporaine de l'organisme comme système d'information. 65

Cette étude fait néanmoins un lien théorico-méthodologique qui signale d'autres domaines de défis pour l'actualisation critique du cadre de Canguilhem. Il s'agit, de la même manière qu'est recherché dans Épidémiologie et émancipation le sens de l'action politique derrière les structures normative-paradigmatiques, rechercher aussi les interactions que conforment les perspectives subjectives identifiées dans cette action politique. Avec d'autres mots, on a cherché élucider dans les propositions qui ont validé les discours épidémiologiques les prétentions, les conditions et les exigences de validité qui ont construit son effectivité communicationnelle et sa légitimation des sujets et des institutions que la soutenaient. Sur la base de l’herméneutique de Gadamer $^{66}$ et Ricœur ${ }^{67}$, mais spécialement sur la Théorie de l'Action Communicative de Habermas, ${ }^{68}$ il a été possible de dépasser ce qui peut être aussi un aspect du cadre de l'épistémologie historique qui doit être reconstruit de façon critique. S’il est vrai que Canguilhem considère les interactions sociales comme déterminantes sur ce que fait un scientifique dans "son laboratoire", c'est encore cet "individu” scientifique, porteur d'une raison qui cherche des réponses aux problèmes mis en place par sa science, qui produira les concepts. Ne serait-ce pas un trait solipsiste qui perdure comme un obstacle aux réponses que les temps nouveaux de la Santé Collective ont besoin d'obtenir de l'épistémologie historique? Ne serait-il pas nécessaire découvrir dà quel dialogue nous participons quand nous faisons une science pour pouvoir en effet apprendre les prétentions/conditions/exigences normatives qui nous orientent comme ses sujets?

Enfin, dans la seconde ligne déployée de la TPTS nous voyons surgir la nécessité d'un autre Canguilhem, pas tellement l'historien des sciences, mais plutôt le philosophe de la médecine. La critique réalisée ici au paroxysme techniciste des pratiques d'attention à la santé a été dirigée par diverses motivations reconstructives en interrelation - nécessité d'une attention intégrale à la santé, humanisation des pratiques, respect des sujets dans leur diversité et leurs droits synthétisées dans le concept de Soin. ${ }^{69}$ Le dialogue ainsi restreint pratiquement au Canguilhem du Le normal et le pathologique, qui en actualisant la maxime de Leriche "La santé est la vie dans le silence des organes", nous apprend que l'expérience de tomber malade est toujours perçue comme un obstacle à un modèle normatif antérieur et qui précède ontologiquement sa tradition dans la morpho-physiologie. C'est celui qui nous montre aussi que la maladie n'est pas un excès ou un manque de quelque chose que nous avons dans la "normalité", mais une différence et de nouvelles possibilités.

Toutefois, dans la plupart de mes travaux en relation au Soin, la préoccupation principale s'est faite autour des caractéristiques des interactions entre les professionnels de santé et les utilisateurs des services et, dans ce sens, la conception de la maladie (et de la santé), directement tributaire de Canguilhem, comme indiqué ci-dessus, demande à être mieux explorée. Il y a à peine un travail sur lequel j'avancé un peu plus dans ce sens: Une conception herméneutique de la santé.70 Ici la

${ }^{62} \mathrm{G}$. Canguilhem, Ideologia e racionalidade nas ciências da vida.

${ }^{63} \mathrm{G}$. Canguilhem, O conbecimento da vida.

${ }^{64} \mathrm{G}$. Canguilhem, O normal e o patológico.

${ }_{65}$ G. Canguilhem, "Epistemology of biology" ["Epistémologie de la biologie"]. In: A vital rationalist - selected writings from Georges Canguilhem [Un rationaliste vitaliste - écrits sélectionnés de Georges Canguilhem], ed. F. Delaporte (New York: Zone Books, 1994), 67-90.

${ }^{66}$ H. G. Gadamer, Verdade e método: traços fundamentais de uma bermenêutica filosófica.

${ }^{67} \mathrm{P}$. Ricoeur, Del texto a la acción: ensayos de hermenêutica II [Du texte à la action: essais d'berméneutique II] (Buenos Aires: Fondo de Cultura Económica, 2010).

${ }^{68} \mathrm{~J}$. Habermas, Teoria do agir comunicativo [Théorie de l'agir communicationnel] (São Paulo: Martins Fontes, 2012).

${ }^{69}$ J. R. C. M. Ayres, Cuidado: trabalho e interação nas práticas de saúde [Soin: le travail et l'interaction dans les pratiques de sante] (Rio de Janeiro: CEPESC; IMS/UERJ; Abrasco, 2011).

${ }^{70}$ J. R. C. M. Ayres, "Uma concepção hermenêutica de saúde". 
référence à Canguilhem ${ }^{71}$ est plus explicite et également inspirée par les réflexions de Gadamer, ${ }^{72}$ similaires en de nombreux sens, ceci a été le plus loin que j'ai choisi de cheminer pour répondre au pressant défi de repenser nos conceptions de la santé et de la maladie. J’y défend la différence non seulement de degré ou de qualité entre elles, sinon la diversité de signifié pratique même des expériences auxquelles elles remettent comme expressions discursives. Mais en même temps, j'y défend l'inseparabilité de ces expériences et leur respectives expressions discursives, étant donné que les maladies et les lectures technico-scientifique que nous faisons d'elles sont de puissants interprètes de la compréhension de la santé dans laquelle nous habitons et qui, comme dirait Leriche, circule "dans le silence des organes" (et des préventivistes, ajouterais-je).

Un résultat de cette conception de la santé est que la médecine, la Santé Collective, les pratiques de santé d'une manière générale, doivent réaliser au-delà et conjointement de toute construction objectivante de la maladie, une vraie "herméneutique" de la santé, et nous, étudiants et professionnels de la santé, nous devons être ses interprètes attentionnés. À ma satisfaction j’ai rencontré récemment une proposition assez similaire chez Canguilhem. Je laisse alors à Canguilhem les derniers mots de cet article qui, finalement, n'est seulement qu'une partie d'un grand dialogue dont nous souhaitons qu'il continue.

La vérité de mon corps, sa constitution même ou son authenticité d'existence, n'est pas une idée susceptible de représentation (...) Cette santé sans idée, à la fois présente et opaque, est pourtant ce qui supporte et valide, en fait et en dernier ressort, pour moi-même, et aussi bien pour le médecin en tant qu'il est mon médecin, ce que l'idée du corps, c'est-à-dire le savoir médical, peut suggérer d'artifices pour la soutenir. Mon médecin, c'est celui qui accepte, ordinairement, de moi que je l'instruise sur ce que, seul, je suis fondé à lui dire, à savoir ce que mon corps m'annonce à moi-même par des symptômes dont le sens ne m'est pas clair. Mon médecin, c'est celui qui accepte de moi que je voie en lui un exégète avant de l'accepter comme réparateur ${ }^{73}$

\section{Remerciement}

A la professeur Maria Novaes pour l'aide généreuse dans l'accès de quelques sources bibliographiques et informations sur la période de Sergio Arouca et le groupe de l'Unicamp des années 1970, et au professeur Ricardo Teixeira pour son examen attentif et généreux de la version finale en français.

\footnotetext{
${ }^{71}$ Georges Canguilhem, $O$ normal e o patológico.

${ }^{72}$ H. G. Gadamer, The enigma of health: the art of healing in a scientific age [Pbilosophie de la sante] (Stanford: Stanford University Press, 1996).

${ }^{73} \mathrm{G}$. Canguilhem, Escritos sobre a medicina, 45.
} 